\title{
REVIEW ARTICLE \\ The multiple facets of the Golgi reassembly stacking proteins
}

\author{
Fabian P. VINKE ${ }^{\star} \dagger$, Adam G. GRIEVE ${ }^{*} \dagger$ and Catherine RABOUILLE ${ }^{*}{ }^{1}$ \\ ${ }^{*}$ Cell Microscopy Centre, Department of Cell Biology, UMC Utrecht, AZU H02.313, Heidelberglaan 100, Utrecht, The Netherlands and †Hubrecht Institute for Developmental Biology \\ and Stem Cell Research, Uppsalaan 8, 3584 CT Utrecht, The Netherlands
}

\begin{abstract}
The mammalian GRASPs (Golgi reassembly stacking proteins) GRASP65 and GRASP55 were first discovered more than a decade ago as factors involved in the stacking of Golgi cisternae. Since then, orthologues have been identified in many different organisms and GRASPs have been assigned new roles that may seem disconnected. In vitro, GRASPs have been shown to have the biochemical properties of Golgi stacking factors, but the jury is still out as to whether they act as such in vivo. In mammalian cells, GRASP65 and GRASP55 are required for formation of the Golgi ribbon, a structure which is fragmented in mitosis owing to the phosphorylation of a number of serine and threonine residues situated in its $\mathrm{C}$-terminus. Golgi ribbon unlinking is in turn shown to be part of a mitotic checkpoint. GRASP65 also seems to be the key target of signalling events leading to reorientation of the Golgi during cell migration and its breakdown during apoptosis. Interestingly, the Golgi ribbon is not a feature of lower eukaryotes, yet a GRASP homologue is present in the
\end{abstract}

genome of Encephalitozoon cuniculi, suggesting they have other roles. GRASPs have no identified function in bulk anterograde protein transport along the secretory pathway, but some cargospecific trafficking roles for GRASPs have been discovered. Furthermore, GRASP orthologues have recently been shown to mediate the unconventional secretion of the cytoplasmic proteins AcbA/Acb1, in both Dictyostelium discoideum and yeast, and the Golgi bypass of a number of transmembrane proteins during Drosophila development. In the present paper, we review the multiple roles of GRASPs.

Key words: cis-Golgi membrane protein of $130 \mathrm{kDa}$ (GM130), functional organization, Golgi ribbon, Golgi stack, Golgi reassembly stacking protein (GRASP), mitotic checkpoint, mitotic phosphorylation PDZ, trans-oligomerization, unconventional secretion.

\section{INTRODUCTION}

\section{Identification of GRASP65 (Golgi reassembly stacking protein) and GRASP55 as Golgi-stacking factors}

The Golgi apparatus is comprised of flattened membrane-bound compartments called cisternae, which are apposed to one another to form a Golgi stack (Figure 1A). In mammalian cells, these stacks (typically comprising three to four cisternae with a cross-sectional diameter of $1 \mu \mathrm{m}$ [1]) are laterally linked by tubules forming the Golgi ribbon, normally localized around the juxtanuclear microtubule-organizing centre [2,3]. In lower eukaryotes, the basic organization of stacked Golgi cisternae is observed, but there are differences compared with the mammalian Golgi. For instance, in Saccharomyces cerevisiae only $40 \%$ of the cisternae are in stacks and in both Pichia pastoris and Drosophila melanogaster Golgi stacks are clearly present, but are not linked into Golgi ribbons [4-6]. Instead, individual stacks are localized next to tER [transitional ER (endoplasmic reticulum)] sites [equivalent to ERES (ER exit sites)] and form tER-Golgi units [5]. The last order of complexity is that the Golgi stacks are polarized with the cis-cisterna close to the entry face of the Golgi and the trans-cisterna close to the exit face.

In view of the dynamic nature of the Golgi apparatus, how such an organized structure is generated and maintained remains largely unexplained. Indeed, it continuously receives and dispatches a flux of newly synthesized proteins and lipids coming from the ER en route to the plasma membrane and the extracellular medium, as well as being involved in the synthesis of many lipid species. The Golgi combines these functions with receiving input from the endosomal system, as well as sending material back to the ER to be re-used. However, under physiological conditions, Golgi stacks are visible and can be isolated, suggesting the existence of specific mechanisms ensuring the maintenance of this unique architecture.

In order to characterize molecules important for maintaining Golgi stacking, different approaches have been taken. First, Cluett and Brown [7] showed the presence of a proteinaceous structure responsible for the cisternal cross-linking of the Golgi stack by treating isolated rat liver Golgi stacks with an array of proteases that resulted in their disassembly into individual cisternae. Triton $\mathrm{X}-100$ solubilization of isolated Golgi stacks in low salt yielded the isolation of the so-called 'Golgi matrix' [8]. Antisera raised against this matrix led to the identification GM130 (cis-Golgi membrane protein of $130 \mathrm{kDa}$ ), one of the first Golgi structural proteins to be discovered [9].

A second approach made use of cell-free assays mimicking the disassembly and reassembly of the Golgi apparatus at the onset and exit of mitosis. At the onset of mitosis, the mammalian Golgi complex breaks down into clusters of tubules and vesicles that partition into two daughter cells to form a new Golgi at telophase

\footnotetext{
Abbreviations used: BMCC, 1-biotinamido-4-[4'-(maleimidoethyl-cyclohexane)carboxy-amido]butane; CD8, cluster of differentiation 8; COPI, coatamer protein I; COPII, coatamer protein II; CDK1, cyclin-dependent kinase 1; dGRASP, Drosophila GRASP; ER, endoplasmic reticulum; ERES, ER exit site; ERK, extracellular-signal-regulated kinase; FRAP, fluorescence recovery after photobleaching; GFP, green fluorescent protein; Gly ${ }^{2}$, glycine residue at position 2; GM130, cis-Golgi membrane protein of 130 kDa; GRASP, Golgi reassembly stacking protein; MAPK, mitogen-activated protein kinase; MEK1, MAPK/ERK kinase 1; NEM, N-ethylmaleimide; NRK, normal rat kidney; Plk1, Polo-like kinase 1; RNAi, RNA interference; siRNA, short interfering RNA; SH3, Src homology 3; SPR, serine/proline-rich; tER, transitional endoplasmic reticulum; TGF $\alpha$, transforming growth factor $\alpha$; VSV-G, vesicular-stomatitis virus G protein.

1 To whom correspondence should be addressed (email c.rabouille@umcutrecht.nl).
} 


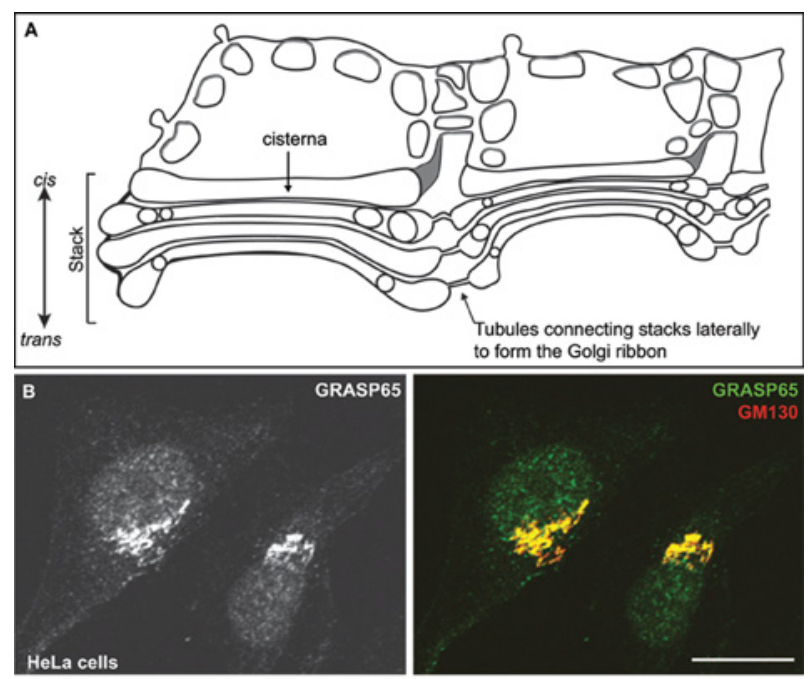

Figure 1 Golgi apparatus and GRASP65 localization

(A) Schematic representation of Golgi stacked cisternae (in a cis-to-trans fashion) laterally linked together by small tubules to form a Golgi ribbon. (B) GRASP65 Iocalization in mammalian HeLa cells by immunofluorescence (green) using a polyclonal antibody to GRASP65 (a gift from Adam Linstedt) showing co-localization with its Golgi receptor GM130 (red). Scale bars, $10 \mu \mathrm{m}$.

$[10,11]$. This cycle was reproduced in a cell-free assay using isolated rat liver Golgi stacks treated with mitotic cytosol yielding disassembled Golgi membrane (called mitotic Golgi fragments) [12]. These fragments were re-isolated and then subsequently incubated with interphase cytosol, leading to the reassembly of Golgi stacks [1].

Treatment of intact Golgi stacks with the alkylating agent NEM ( $N$-ethylmaleimide) did not affect the in vitro cycle of disassembly/reassembly. However, when the drug was added after the disassembly step, cisternae would form but did not stack [1]. This suggests that the disassembly exposed a NEM-sensitive factor involved in stacking. This was the basis of the GRASP65/ GRASP55 discovery. Barr et al. [13] adapted the system and used NEM to alkylate all available thiol groups on the intact Golgi stacks, followed by their disassembly with mitotic cytosol. The mitotic Golgi fragments were then incubated with the biotin-tagged NEM-derivative BMCC \{1-biotinamido4-[4'-(maleimidoethyl-cyclohexane) carboxy-amido]butane $\}$ that reacted with the newly exposed thiol groups.

Three BMCC-modified proteins were subsequently identified by streptavidin overlay and MS analysis. One was a protein $65 \mathrm{kDa}$ in size that was named GRASP65 [13]. Its role in stacking was demonstrated further using the disassembly/reassembly cellfree assay, where the function of the endogenous GRASP65 was blocked by addition of antibody or excess of recombinant GRASP65. No Golgi stacks were formed, only single cisternae and vesicles.

The identification of GRASP65 led to that of GRASP55, which is encoded by a different gene but with a very similar role and distribution [14]. The human GRASP55 gene (on chromosome 2 ) is believed to have arisen through the duplication of a region within chromosome 3, which contains GRASP65 [15]. It was then proposed that both proteins are required for Golgi stack formation. As other non-related proteins have also been named GRASP [such as the brain-specific GRIP (glutamate receptorinteracting protein)-associated protein GRASP1], GRASP65 and GRASP55 are often referred in gene and protein databases as GORASP1 and GORASP2 respectively.

\section{GRASP65 and GRASP55 localize to Golgi stacks}

The proposed role of GRASP65 and GRASP55 in Golgi stacking was strengthened further by their subcellular localization. Using immunofluorescence microscopy of HeLa cells, both proteins were found to be restricted to the Golgi complex [13] (Figure 1), but the use of immuno-electron microscopy revealed a slight differential localization. GRASP65 is found more prominently at the cis-Golgi, whereas GRASP55 is localized to the medial- and trans-Golgi cisternae [16].

\section{GRASPs across species}

The sequencing of the genomes of many different species has allowed the identification of GRASP orthologues and homologues in all animal species except plants (see below). These orthologues and homologues have been well described [17] and are present in zebrafish, Xenopus, Drosophila, Anapholes, Apis, Caenorhabditis, Dictyostelium, Trypanosoma and also in $S$. cerevisiae and $P$. pastoris as well as being found in a eukaryote with a much smaller genome Encephalitozoon cuniculi [18]. In addition, Plasmodium falciparum has two isoforms [19].

When tested, most of these GRASPs are found localized to the Golgi complex, for example in Drosophila [20], Trypanosoma [21] and Plasmodium [19,22]. However, a subset is also detected on a wider range of membranes than that found for GRASP65. For instance, the single Drosophila GRASP orthologue dGRASP is also present on ERES and on the ER itself [20]. Grh1, the single GRASP orthologue of the budding yeast $S$. cerevisiae, an organism that has only $40 \%$ of its cisternal membrane stacked, is also found on the tER sites where it binds to COPII (coatamer protein II) vesicles [23]. Grh1 in $P$. pastoris strictly localizes to tER sites, not the Golgi stack [6]. Interestingly, GRASP65 was also observed in the cisGolgi network [24] at ER exit sites of FRT (Fisher rat thyroid) cells [25]. The function of GRASP at ERESs remains to be established, although it has been proposed to facilitate anterograde transport.

\section{THE DOMAINS OF GRASPS}

The identification of GRASP homologues and orthologues in many species has facilitated the extraction of a number of conserved features which are listed in these two articles [26,27]. Essentially, all GRASPs are built up of two regions. First, an N-terminal half (189-208 amino acids, with the exception of yeast) also called the GRASP domain and contains two putative PDZ domains [17]. The second region is the C-terminal half which is not conserved between species but is rich in proline and serine residues, as well as glutamine and asparagine residues (Figure 2).

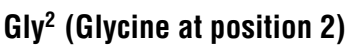

Although GRASPs do not contain any membrane-spanning domains, they are tightly associated with Golgi membranes. Anchoring occurs via N-myristoylation of $\mathrm{Gly}^{2}$, at least for mammalian and Drosophila GRASPs $[13,14,20]$. Gly ${ }^{2}$ is also conserved across species, except in yeast and one splice variant of Plasmodium (Figure 2). Indeed, budding yeast use an acetylated amphipathic helix and, interestingly, the malaria parasite $P$. falciparum can use both $\mathrm{N}$-myristoylation-dependent and -independent targeting, owing to alternative splicing of the GRASP N-terminus [19,23]. 


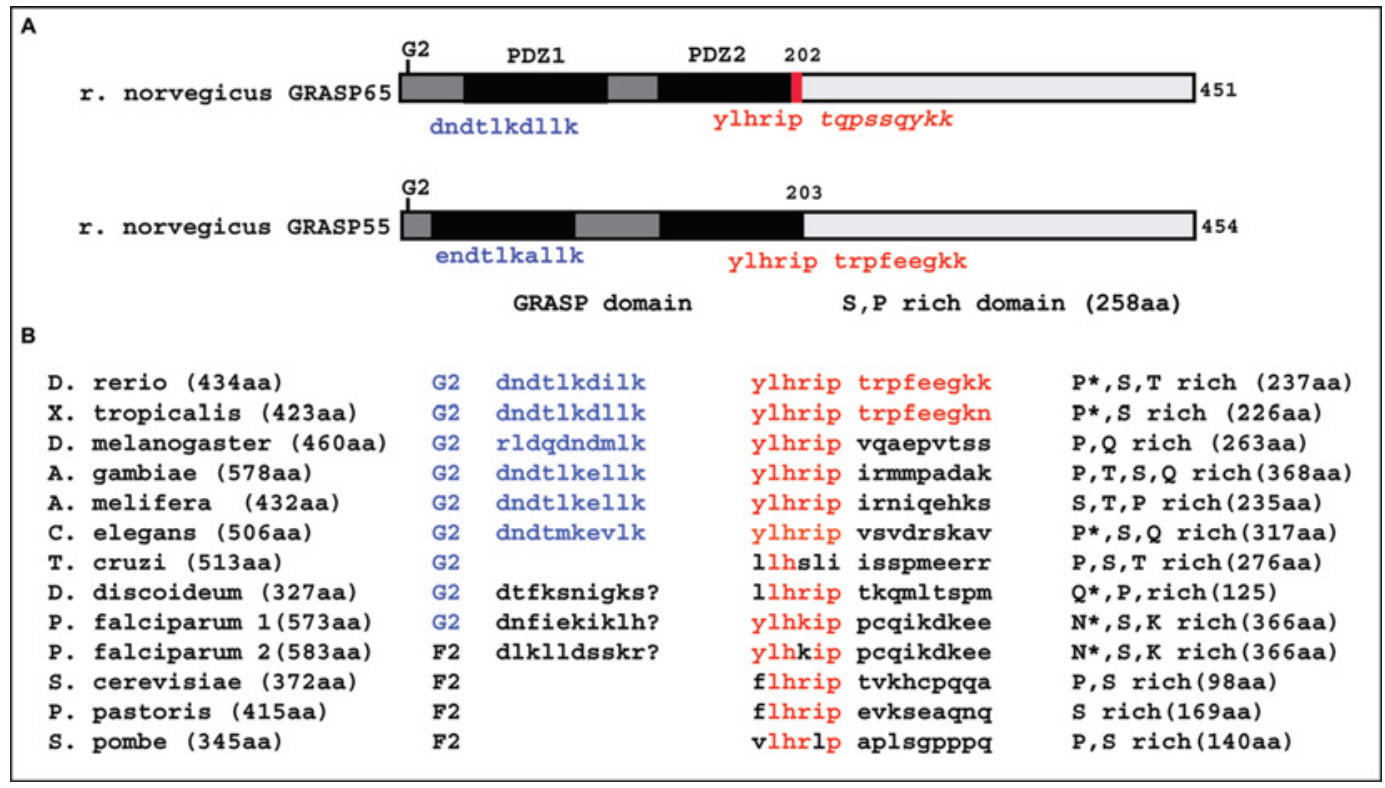

Figure 2 Functional domains of the GRASP homologues and orthologues

(A) Schematic representation of rat GRASP65 (AF015264) and GRASP55 (AF110267) proteins. The N-terminal half contains the two putative PDZ domains, the trans-oligomerization domain (blue) being nested in the first one. The GM130-binding domain is represented in red italics. The C-terminus is rich in serine and proline residues. (B) Comparison between species of the GRASP length, the conservation of the Gly², trans-oligomerization domain and the GM130-binding domain, the C-terminus enrichment in amino acids and its length in D. rerio (NP_956997 NCBI); Xenopus tropicalis (AAH74543 NCBI); D. melanogaster (AAF49092 NCBI); A. gambiae (PP_308450 NCBI); A. melifera (PP-393076 NCBI); C. elegans (NP_501354 NCBI); T. cruzi (EAN99074 NCBI); D. discoideum (EAL60823 NCBI); P. falciparum GRASP1 (XP_002585405 NCBI) and GRASP2 (XP_001347453 NCBI); S. cerevisiae (NP_01805 NCBI); P. pastoris (proprietary P. pastoris genome sequence from Integrated Genomics) and S. pombe (NP_593015 NCBI). * represents amino acids enriched 4-fold or more above random.

\section{The Golgi receptor of GRASP65}

Although the Golgi localization of GRASP65 requires the myristoylation of $\mathrm{Gly}^{2}$, it is also strictly dependent on its interaction with a Golgi receptor, identified as the coiled-coil Golgin GM130 [14,28]. The region of GRASP65 required for this interaction is mapped to a stretch of amino acids (in rat $\mathrm{T}^{189}$ QPSSQYKK ${ }^{201}$ is present in the second putative PDZ domain; Figure 2) that binds the motif XXNDXXXIMVI (where $\mathrm{X}$ is any amino acid) present at the C-terminus of GM130. Furthermore, using GFP (green fluorescent protein)tagged truncated chimaeras, both domains were found to be essential for Golgi localization [28]. Initially, GRASP65 was proposed to be the receptor of GM130, but GRASP65 knockdown by siRNA (short interfering RNA) does not change GM130 localization, whereas depletion of GM130 results in a loss of GRASP65 from the Golgi membrane [28-30]. This suggests that GM130 is the receptor for GRASP65. Of note, the recent depletion of both GRASP55 and GRASP65 resulted in a decrease in GM130 staining in the Golgi, perhaps owing to the resulting damage to the Golgi stack structure [31].

\section{The Golgi receptor of GRASP55?}

Aligning the GM130-binding region of GRASP65 with GRASP55 reveals an almost identical motif (Figure 2) [26]. Furthermore, GRASP55 can bind GM130 but with lower affinity than GRASP65 [32]. However, GRASP55 preferably binds to the C-terminus of Golgin45, a putative Golgi matrix protein, mirroring GRASP65 binding to GM130. Golgin 45 could therefore be the GRASP55 Golgi receptor, although this remains to be proven. Indeed, knockdown of Golgin 45 by RNAi (RNA interference) severely disrupted the Golgi ribbon, establishing the role of this protein in Golgi organization, but the effect on GRASP55 was not assessed [32]. Interestingly, GRASP55 also binds Rab1 and Rab2, which could possibly mediate its recruitment to the early secretory pathway.

The GM130-binding motif is not conserved (Figure 2), although the five amino acids immediately upstream of it are. However, in S. cerevisiae, Grh1 binds Bug1, a protein structurally related to GM130 and, in Drosophila S2 cells, dGRASP interacts genetically with GM130 in the maintenance of the Golgi stack. Whether GM130 orthologues act as receptors for GRASP and by which type of interaction remains to be established.

\section{PDZ domains and trans-oligomerization}

The N-terminal half of GRASP is largely conserved and comprises two putative PDZ domains which can mediate interactions with other proteins and lipids, yet to be determined [17]. These domains remain to be confirmed as bona fide PDZ domains, which are defined by secondary structure rather than by amino acid sequence similarity. This issue could be partially resolved by solving the crystal structure of the GRASP domain.

In GRASP65, the first PDZ domain contains a small stretch of amino acids necessary for trans-oligomerization (Figure 2). This molecular property was first demonstrated by the ability of GRASP65 to cross-link beads [26,33]. In vitro experiments indicate that GRASP65 forms stable cross-bridges with itself between adjacent Golgi cisternae [14]. This property has also been observed in vivo, as ectopic localization of GRASP to mitochondria led to their tethering to one another. Thus GRASP65 and GRASP55 have the potential to connect different subsets of Golgi membranes that could be accomplished via their homotypic trans-oligomerization [34,35]. These domains are conserved in Drosophila, other insects, zebrafish, Xenopus and worms, 
perhaps in Dictyostelium and Plasmodium, but do not seem to be present in Trypanosoma and yeasts.

\section{C-terminal half}

In GRASP65 and GRASP55, the less conserved C-terminal region contains an SPR (serine/proline-rich) domain that can be phosphorylated at several sites and plays an important role during mitosis. Interestingly, the C-termini of GRASPs across species (which are variable in length) are rich in proline and serine/threonine residues but also in some cases are very rich in glutamine and asparagine residues (Figure 2). Proline-rich motifs are known substrates of SH3 (Src homology 3) domains [36]. Given the high proportion of proline-rich motifs in the Ctermini of GRASPs, it could interact with SH3-domain-containing partners, giving a future avenue for investigations into the GRASP C-terminus.

\section{THE FUNCTION OF GRASPS IN GOLGI ARCHITECTURE}

\section{GRASPs and Golgi stacking in vivo}

The results obtained in vitro with the cell-free assay described above strongly indicated that both GRASP65 and GRASP55 were stacking factors, but what about in vivo? To address this issue, two types of experiment have been performed leading to conflicting results.

Interfering with GRASP function by micro-injection of an anti-GRASP65 inhibitory antibody in NRK (normal rat kidney) cells in metaphase (in which the Golgi is disassembled) resulted in disruption of Golgi stack formation when cells exited mitosis. Instead, tubulo-reticular networks, fenestrated cisternallike structures and an increase in vesicular, often coated, structures around the cisternae were observed. These phenotypes were accompanied by the appearance of misaligned stacked cisternae, suggesting that GRASP65 was required for Golgi stacking $[33,37]$.

On the other hand, depleting GRASP65 from HeLa cells by siRNA did not result in a change in Golgi localization and organization when assessed by immunofluorescence [29,30]. Quite strikingly, the Golgi stacks remained largely intact, although their cross-sectional diameters were slightly, but significantly, larger and the resulting stacks contained fewer cisternae per stack (two to three compared with three to four in mock-depleted cells) [29].

GRASP55 depletion from HeLa cells did not seem to affect Golgi stacking either, when assessed by both light and electron microscopy. The number of Golgi stacks per cell section and their cross-sectional diameter were unaltered $[38,39]$. However, a more recent study has reported a small, but significant, reduction in the number of cisternae per stack (from five to six cisternae in mockdepleted HeLa cells to three to four cisternae after GRASP55 depletion) [31].

However, double depletion of GRASP65 and GRASP55 had a more profound effect on stacking. The Golgi pattern observed by immunofluorescence was unchanged but ultrastructural examination by electron microscopy revealed the absence of stacked cisternae in over $80 \%$ of the cells, illustrating for the first time a role for GRASP65 and GRASP55 in Golgi stacking in vivo in mammalian cells [31]. This also suggests that for stacking GRASP65 and GRASP55 provide redundancy for one other.

Although in HeLa cells the issue could be perceived as momentarily resolved, depletion or knockout mutants in lower eukaryotes where only one GRASP protein is present, such as in T. brucei (Chris de Eraffenried and Graham Warren [6]) and
P. pastoris personal communication, did not lead to any obvious loss of stacking [6]. In Drosophila S2 cells, only $20 \%$ of the Golgi stacks were affected and the effect was much stronger when their single GRASP was co-depleted with the Drosophila GM130 homologue [20]. Furthermore, GRASP is present on Golgi membranes where no stacks are visible, such as in $S$. cerevisiae and in certain stages of Drosophila development, even though these Golgi apparatuses are completely functional, at least for transport and glycolysation $[5,23,40]$. There must also be other stacking factors not related to GRASPs, as plants display Golgi stacks but no plant GRASP homologues have been identified so far [17].

\section{Building the Golgi ribbon}

Although, as mentioned above, single depletion of either GRASP65 [29,30] or GRASP55 [39] does not lead to a very strong effect on Golgi stacking, it does strongly affect Golgi organization, as the Golgi stacks are no longer found to be connected into a characteristic ribbon. This was shown not only by electron microscopy, but also by FRAP (fluorescence recovery after photobleaching) experiments where a small portion of the Golgi ribbon, decorated with GFP-tagged Golgi-resident enzymes, was bleached and did not recover as fully as in nondepleted cells $[30,39]$. This led to the hypothesis that GRASP65 and GRASP55 are required for lateral fusion of the Golgi cisternae during Golgi ribbon formation, but in a non-redundant manner, as single depletion leads to Golgi ribbon unlinking. The role of GRASP65 in linking cisternae has been shown to be mediated by binding to GM130 and it is possible that GRASP55 employs its preferred binding partner, Golgin45, to fulfil a similar role to GM130 [30,32,41].

It is noteworthy that GRASP55 depletion was also performed by Duran et al. [38] and was not found to play a role in Golgi ribbon formation or maintenance, assessed either by electron microscopy or FRAP. The jury is therefore still out regarding the role of GRASP55 in Golgi ribbon linking but GRASP65 seems to convincingly have a function in it. Golgi ribbons, however, are mainly found in mammalian cells and are almost absent in lower organisms where a GRASP orthologue is clearly present and functional. In fact, it seems that most organisms that show clear ribbons have two GRASPs, whereas those that have one GRASP have ministacks (or no stack at all). This strongly suggests other roles for these proteins.

\section{Trans-oligomerization: a mechanism for cisternal stacking and Golgi ribbon formation}

As discussed above, GRASP65 and GRASP55 are involved in the stacking of Golgi cisternae and ribbon formation and this property could be conferred by their ability to trans-oligomerize. The first evidence for trans-oligomerization was gathered from the observation that GRASP65-coated beads aggregate [26]. This was shown to be mediated by the GRASP N-terminal region that contains two putative PDZ domains. The transoligomerization domain location was narrowed down to the first 112 amino acids of GRASP65, thus mainly confined to PDZ1 and then further to the stretch of 11 amino acids described in Figure 2 [34,42]. Secondly, expressing GRASP65 fused to a mitochondrial-targeting domain leads to mitochondrial clustering in vivo, suggesting that GRASP65 is able to tether adjacent membranes in a trans configuration [34]. This work also identified the importance of the putative PDZ1 groove, that strongly argues that the physical basis for tethering is a PDZ interaction with an unknown ligand. This ligand has been recently identified as the GRASP65 PDZ2 domain (comprising the 20 amino acids 


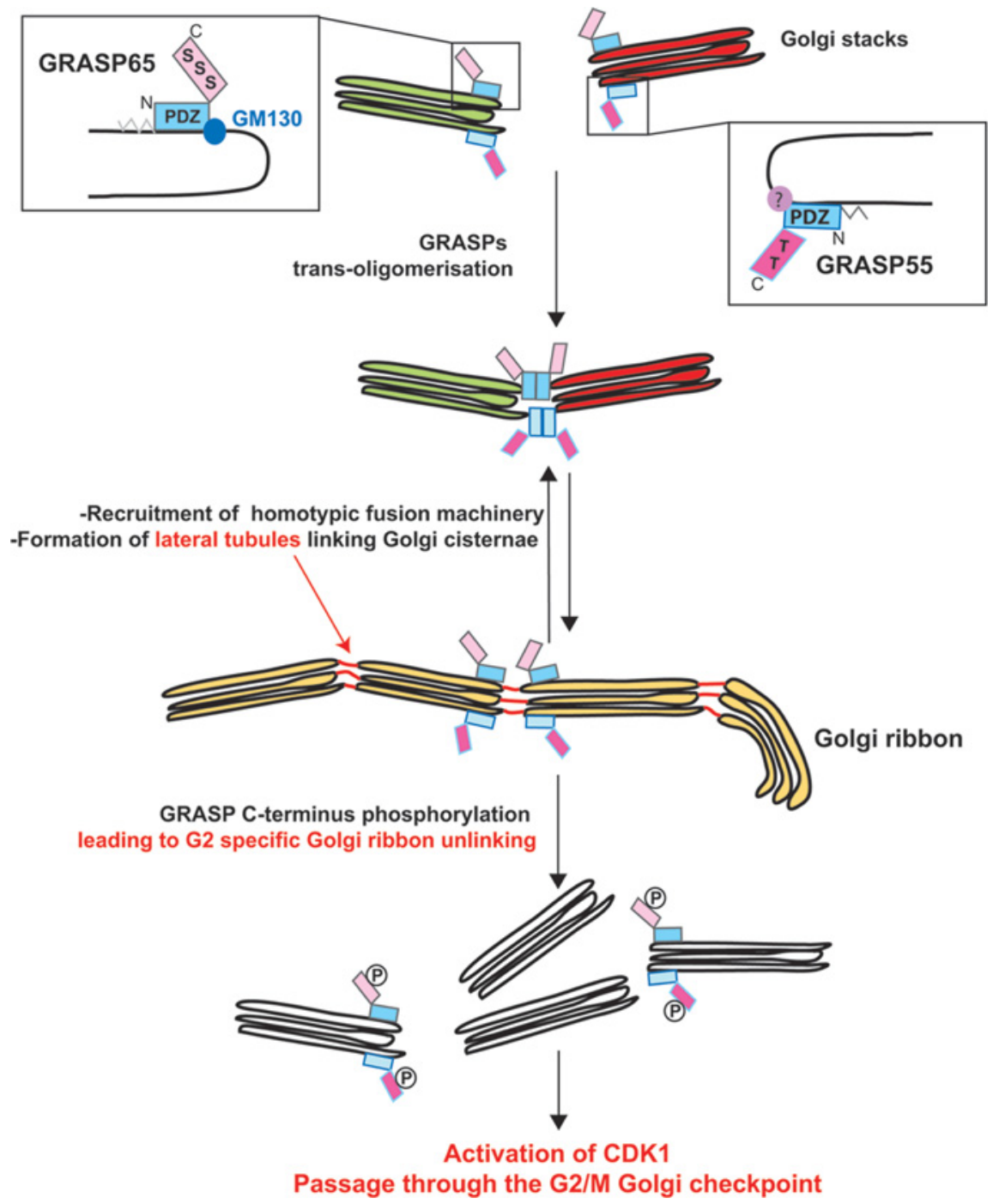

Figure 3 Modulation of GRASP trans-oligomerization at the onset of mitosis

GRASP55 and GRASP65 are tightly bound to the Golgi apparatus via the myristoylated N-terminus (grey zigzag in the inset) and their interaction with GM130 for GRASP65 and p0ssibly Golgin45 for GRASP55. Both proteins harbor PDZ domains in their N-terminal half that mediate GRASP trans-oligomerization thus facilitating subsequent homotypic fusion of Golgi stacks into the Golgi ribbon through the formation of lateral tubules. These tubules allow the diffusion of proteins and lipids across the ribbon which are shown by the mixing of green and red colour into yellow. We propose that once tubule formation is achieved, GRASP trans-oligomerization no longer takes place, but can be triggered again when the tubules are remodelled, for instance during protein transport or Golgi re-positioning. At the $\mathrm{G}_{2}$-phase of the cell cycle, the C-terminal half of GRASP65 and GRASP55 is phosphorylated by MEK1/ERK at Thr ${ }^{225}$ and Thr ${ }^{249}$ and CDK1 and PIk1 at Ser ${ }^{216} / \mathrm{Ser}^{217}$, Ser ${ }^{189}$, $\mathrm{Thr}^{220}$, $\mathrm{Ser}^{277}$ and $\mathrm{Ser}^{376}$ ), leading to the inhibition of GRASP trans-oligomerization resulting in Golgi ribbon unlinking, putative activation of $\mathrm{CDK1}$ and passage through the $\mathrm{G}_{2} / \mathrm{M}^{\mathrm{G}} \mathrm{Golgi}$ checkpoint.

between Cys ${ }^{192}$ and Lys $^{212}$ ) of an adjacent GRASP molecule [43]. For optimal trans-oligomerization-mediated membrane tethering, GRASPs need to be anchored to a membrane at two points (e.g. via myristoylation at $\mathrm{Gly}^{2}$ and via PDZ2 binding to GM130), thus allowing the protein to lie parallel to the membrane (Figure 3) [35]. Expression of the 112 amino acid domain in HeLa cells increased the average number of cisternae per stack (shown by electron microscopy), thus illustrating the relevance of this tethering ability to Golgi organization in vivo [42]. In theory, GRASP55 and GRASP65 should trans-oligomerize as their PDZ domains are very similar, but this has not yet been resolved.

Interestingly, the trans-oligomerization domain does not seem to be present in lower organisms where no stacks are present. Whether the trans-oligomerization properties correlate with the presence of stacks remains to be investigated, perhaps by testing the in vitro ability of GRASPs, across species, to form stacks or at least cross-link beads.

\section{MODULATION OF THE GOLGI ARCHITECTURE BY GRASP MITOTIC PHOSPHORYLATION AT THE ONSET OF MITOSIS: A $_{2} / \mathrm{M}$ CHECKPOINT?}

As mentioned above, the C-terminal halves of GRASP65 and GRASP55 do not share the same high amino acid identity as their N-termini. Nevertheless, they are both rich in proline and serine/threonine residues, with specific serine and threonine residues shown to be mitotically phosphorylated. These phosphorylation events lead to Golgi disassembly observed at the onset of mitosis, characteristically following the pattern of Golgi 
ribbon breakdown into Golgi stacks (referred to as Golgi ribbon unlinking), and then subsequent fragmentation of the Golgi stacks into vesicles and tubules leading to what is known as mitotic Golgi clusters.

\section{GRASP65 and GRASP55 phosphorylation}

GRASP65 is a major mitotic Golgi phosphoprotein [13,33,44]. Four serine/threonine residues $\left(\mathrm{Ser}^{216} / \mathrm{Ser}^{217}, \mathrm{Thr}^{220}, \mathrm{Ser}^{277}\right.$ and $\mathrm{Ser}^{376}$ ) have been identified to be targeted by the key mitotic kinase CDK1 (cyclin-dependent kinase 1) [45]. Two other phosphorylated serine residues were also identified, but the kinases responsible were not. GRASP65 is also a Plk1 (Polo-like kinase 1) substrate $[33,44,46]$. Plk1 was shown to dock on to CDK1-phosphorylated GRASP65, suggesting it could be recruited on Golgi membrane and drive the fragmentation of the Golgi complex [45]. Furthermore, in addition to CDK1, GRASP65 has been speculated to also be a substrate for MEK1 [MAPK (mitogen-activated protein kinase)/ERK (extracellularsignal-regulated kinase) kinase 1]/ERK. However, despite the fact that ERK phosphorylates GRASP65 at interphase in response to EGF (epidermal growth factor) stimulation (and during cell migration), GRASP65 does not seem to be mitotically phosphorylated by MEK1/ERK [45,47].

Conversely, GRASP55 only has two threonine residues $\left(\mathrm{Thr}^{225}\right.$ and $\mathrm{Thr}^{249}$ ) that are targeted for phosphorylation during mitosis by either ERK2 or ERK1c or both [48,49]. Furthermore, GRASP55 has been scored as a hit in a screen for proteins able to bind Polobox domains in cells arrested in prometaphase and therefore is also potentially phosphorylated by Plk1 [50].

The loss of the Golgi-stacked architecture (either by Golgi ribbon unlinking and/or Golgi fragmentation) upon GRASP65 mitotic phosphorylation is explained by the disruption of the transoligomerization of GRASP65 homodimers [26,33]. Indeed, serine phosphorylation of GRASP65 leads to impairment of GRASP65 dimers to form higher order oligomers [33]. GRASP55 has also been shown to oligomerize in a cell-cycle-dependent manner in vitro. Incubation of GRASP55 with either mitotic cytosol or purified MEK1/ERK2 kinases, thus leading to $\mathrm{Thr}^{225}$ and $\mathrm{Thr}^{249}$ phosphorylation, abolished its oligomerization properties [31]. Whether these phosphorylation events caused Golgi ribbon unlinking and fragmentation to occur in vivo remains to be investigated.

\section{GRASP65 and GRASP55 mitotic phosphorylation drives Golgi ribbon fragmentation: a possible $G_{2} / M$ checkpoint}

Remarkably, preventing GRASP65 or GRASP55 phosphorylation, thus preventing Golgi ribbon unlinking, also resulted in a delay in mitotic entry. This delay was postulated to be due to the inhibition of Golgi fragmentation and led to the notion of a mitotic checkpoint embedded in the Golgi architecture [51,52]. Reinforcing this notion is the finding that expression of the transoligomerization domain of GRASP65 in HeLa cells increased the average number of cisternae per stack (as mentioned above), but also inhibited mitotic Golgi fragmentation and delayed cell-entry of mitosis (Figure 3) [42].

The first observation leading to this novel concept was the inhibition of Golgi fragmentation (as assessed by immunofluorescence microscopy), as well as the failure of cells to proceed through mitosis, upon microinjection of an inhibitory antibody targeting part of the C-terminal region of GRASP65 [53]. However, when Golgi fragmentation was performed prior to injection (by either nocodazole or Brefeldin A treatment, for instance), the block of cell entry into mitosis was alleviated. This suggested that the fragmentation of the Golgi ribbon could serve as a sensor for controlling entry in mitosis in mammalian cells. These results were confirmed by expression of the $\mathrm{C}$-terminal region of GRASP65 that significantly impaired or delayed cell entry into mitosis $[29,45]$. Strikingly, expression of a fully phosphorylationdefective version of the same C-terminal region released this block/delay [45]. These results suggest that phosphorylation of the GRASP65 C-terminus is necessary to induce this Golgi checkpoint.

Similarly, the expression of a GRASP55 ERK1/2 phosphorylation-defective version (T225A, T249A) inhibits Golgi ribbon unlinking and also blocks and/or delays the cells at $\mathrm{G}_{2} / \mathrm{M}$ transition [54]. This delay was also rescued when the Golgi ribbon unlinking and/or fragmentation was pre-induced by GRASP65 depletion [54]. This shows that GRASP65 and GRASP55 non-redundantly function in the mitotic triggering of Golgi ribbon unlinking, which is in turn a requirement for the $\mathrm{G}_{2} / \mathrm{M}$ Golgi checkpoint. Surprisingly, expression of a recombinant C-terminal part of GRASP55, the so-called C100 (amino acids 351-454), which does not contain any mitotic phosphorylation sites, also inhibits Golgi fragmentation and delays entry into mitosis of synchronized cells. The current explanation for this observation is that this region is thought to sequester certain kinases and/or effectors, which might be involved in the phosphorylation of $\mathrm{Thr}^{225}$ or $\mathrm{Thr}^{249}$ [38].

It is, however, unclear how phosphorylation of the C-terminal domain regulates $\mathrm{N}$-terminal domain trans-oligomerization properties that mediate membrane tethering. In this regard, a novel Plk1 site $\left(\operatorname{Ser}^{189}\right.$ ) was very recently identified in GRASP65 and was found to be involved in the $\mathrm{G}_{2}$ Golgi ribbon unlinking. The phosphomimetic S189D abrogated the trans-oligomerization properties of GRASP65 (assessed both using the mitochondria clustering assay or the formation of Golgi ribbon). This serine residue is situated within the PDZ2 domain of GRASP65 and its phosphorylation was shown to impair the interaction between PDZ1 and PDZ2, perhaps by modifying the PDZ2 conformation or its orientation in the membrane, thus preventing tethering. This suggests a model in which the CDK1 phosphorylation event creates a landing platform for Plk1 that in turn phosphorylates the serine residue situated in the GRASP second PDZ domain, thus explaining the link between C-terminus phosphorylation events and Golgi ribbon unlinking at mitosis [43].

As expected, GRASP65 and GRASP55 depletion by RNAi does not prevent mitotic entry, because the resulting Golgi ribbon unlinking allows the cells to pass through the $\mathrm{G}_{2} / \mathrm{M}$ checkpoint $[29,38,39]$. However, GRASP65 depletion leads to an arrest in metaphase with a high percentage of cells displaying aberrant spindles and centrosomal defects [29]. Interestingly, the single $S$. cerevisiae orthologue of GRASP (Grh1), which is non-essential for growth, has been shown to be involved in mitotic progression, since mutant cells fail to arrest upon pharmacological induction of the spindle checkpoint [55].

Overall, these results indicate that the phosphorylation of GRASP65 and GRASP55 may have at least two possible roles on cells progressing into mitosis. The first in severing the tubules linking the Golgi ribbon, thus allowing the cells to pass through the $\mathrm{G}_{2} / \mathrm{M}$ Golgi checkpoint and start mitosis. Their second role may be in Golgi fragmentation, which could be linked directly or indirectly to mitotic spindle organization (see below). However, several aspects of the role of GRASP65 in the Golgi-related checkpoint need further clarification. 


\section{GRASP65 IN SPINDLE FORMATION/REGULATION DURING METAPHASE}

One of the striking features of the mammalian Golgi apparatus is its perinuclear localization and its close proximity to the centrosome, which seems to play an active role in this localization [56-60]. At the onset of mitosis, the centrosome duplicates and forms the spindle poles, which have several roles in dividing the Golgi between each daughter cell [61]. When mitosis is completed, the Golgi is reassembled in a centrosome-dependent manner [62].

Conversely, the Golgi also seems to influence spindle-pole dynamics during mitosis. GRASP65-depleted HeLa cells have multiple, disorganized and non-functional spindle poles and their chromosomes do not align properly on the metaphase plate. As a result, GRASP65-depleted cells do pass the metaphase checkpoint and exit mitosis, and quickly undergo apoptosis [29]. This effect is, however, also observed in GM130-depleted cells, suggesting that spindle formation and maintenance involves the GM130GRASP65 complex [63].

\section{GRASP65 IN APOPTOSIS}

Both in cells entering mitosis and undergoing programmed cell death (apoptosis), the Golgi apparatus disassembles into vesicular and tubular structures, loses its juxtanuclear localization and trafficking through the secretory pathway is inhibited. During apoptosis, however, this process is irreversible [64,65].

Although the morphological changes are similar, the molecular machinery behind Golgi fragmentation during apoptosis is different. The caspases, a class of proteases, are the key players in the apoptotic fate of the Golgi apparatus. Caspase cleavage of a wide array of substrates is one of the main forces that drives apoptosis [66]. Several Golgi proteins, GM130, p115, giantin, syntaxin 5, Golgin160 and GRASP65, are among their targets [67-70] and their cleavage is thought to contribute to Golgi fragmentation. Caspase 3 cleaves GRASP65 at three specific sites present in its C-terminus $\left(\mathrm{Asp}^{320}, \mathrm{Asp}^{375}\right.$ and $\mathrm{Asp}^{393}$ ) and expression of a caspase-resistant form of GRASP65 (where the aspartic acid residues are replaced by alanine Asp393A) inhibited Golgi fragmentation [71]. Furthermore, overexpression of the C-terminal 200 amino acids of GRASP65 prevents Golgi fragmentation and delays apoptosis in neuronal cells [72]. The caspase 3-cleavage sites are not conserved in GRASP55 and whether GRASP55 or other GRASPs are targeted by a caspase for cleavage remains unknown.

\section{GRASP65 IN CELL MIGRATION}

It has become increasingly appreciated that when cells migrate towards a chemotactic cue, the Golgi re-orients towards the leading edge of the migrating cell to provide membrane and proteins required for migration in a targeted manner [73]. Consequently, its structural integrity is important to support cell migration [74]. One of the signalling cascades dictating Golgi reorientation involves ERK-mediated GRASP65 phosphorylation on $\operatorname{Ser}^{277}$ [75]. Indeed, polarization of the Golgi towards the leading edge in response to growth-factor stimulation is blocked by pre-treatment with ERK inhibitors, a result mirrored by expression of a phosphorylation-deficient GRASP65 mutant.

Remarkably, $\operatorname{Ser}^{277}$ is also phosphorylated by CDK1 during mitosis (see above) and this modification causes disassembly of the Golgi complex [45]. Phosphorylation of GRASP65 on $\mathrm{Ser}^{277}$ by ERK also causes disassembly of the Golgi in vitro, but this is not observed in vivo using light microscopy [75]. Nevertheless, undetected local remodelling might modulate Golgi anchoring and/or positioning and promote its orientation, as well as facilitate the intra-Golgi transport probably needed for efficient membranedelivery towards the leading edge. The presence of such a local remodelling event needs to be clarified in order to understand the mechanism of how GRASP65 phosphorylation regulates Golgi orientation.

\section{GRASPS IN PROTEIN TRANSPORT}

\section{No role for GRASPs in bulk secretion}

Classical anterograde ER-Golgi-plasma-membrane protein transport has been described in many reviews recently and is not affected in HeLa and U2OS cells depleted of GRASP65 by RNAi, as monitored either by ER-to-Golgi (KDEL-receptor) or ER-toplasma membrane [using VSV-G (vesicular-stomatitis virus G protein)] transport [29,30,76]. Similar findings are reported for GRASP55 using the secretion of soluble proteins, horseradish peroxidase-oxidase and the deposition of VSV-G [38,39].

In Drosophila S2 cells, depletion of the single GRASP orthologue dGRASP by RNAi did not lead to inhibition of anterograde transport of a reporter protein and cell growth was not affected [20]. It was also not found in two genomewide RNAi screens aimed at identifying factors involved in secretion in Drosophila S2 cells [77,78]. Depletion of GRASP in Dictyostelium, S. cerevisiae and P. pastoris also gives normal cell growth, suggesting that GRASPs do not play a major role in classical-secretion-sustaining growth $[6,17,23,79,80]$.

\section{Facilitation of anterograde transport to and through the Golgi}

However, GRASPs have been proposed to facilitate anterograde transport. In a complex with GM130, GRASP65 has been shown to promote the capture of L-IC (late intermediate compartment) tubular structures (that are positive for both proteins) and allow the entry of cargo in the Golgi. A truncated GM130, G95 (which still contains the GRASP65-binding domain but lacks the p115-binding domain) delays this entry, resulting in impairment of protein secretion [24]. However, this result suggests that the GRASP65-GM130 complex is not essential in this process.

The yeast GRASP Grh1 has been found to interact with the COPII coat proteins Sec23 and Sec24, suggesting that Grh1 could play a role in the docking or fusion of COPII-coated vesicles at the Golgi, thus facilitating anterograde transport through the early secretory pathway [23].

\section{Cargo-specific roles for GRASPs in trafficking and chaperoning}

Finally, GRASP65 has also been proposed to play an indirect role in modulating anterograde transport through its role in Golgi stacking [37]. Injecting mitotic NRK cells with anti-GRASP65 antibodies, or a non-myristoylated form of GRASP65, led to Golgi unstacking and resulted in an increased amount of the transmembrane protein CD8 (cluster of differentiation 8) at the plasma membrane, suggesting that stacking negatively controls the rate of transport. The hypothesis put forward was that COPI (coatamer protein I) vesicles are generated more efficiently when Golgi cisternae are unstacked. To show this, isolated Golgi membranes were unstacked upon GRASP65 phosphorylation by CDK1/cyclinB1 and Plk1, and COPI vesicle budding was initiated by treatment with ARF1 (ADP-ribosylation factor 1) and coatamer as assessed by electron microscopy. The number of 
vesicles formed from unstacked Golgi cisternae was found to be significantly higher than from stacked ones.

In addition, a very small number of proteins have been shown to directly depend on GRASPs for their transport. The secreted factor TGF $\alpha$ (transforming growth factor $\alpha$ ) was found to interact biochemically with GRASP55 in HEK (human embryonic kidney)-293 cells, and GRASP55 seems to act as a chaperone for TGF $\alpha$, mediating transport to the plasma membrane [81]. Disrupting this interaction leads to the retention of TGF $\alpha$ in the Golgi.

Furthermore, several members of the p24 family, namely p24a, TMP21 and gp25L, that are normally involved in ER-to-Golgi trafficking, were also shown to interact with both GRASP65 and GRASP55. p24a has the two C-terminal valine residues that are essential for this interaction. Mutation of these residues to alanine disrupted binding and led to transport of p24a to the cell surface, suggesting that GRASP55 is involved in retaining p24a in the early secretory pathway [41].

Interestingly, $\mathrm{TGF} \alpha$ also has a di-valine motif at its $\mathrm{C}$-terminus leading to the hypothesis of GRASP-dependent transport of Cterminal valine-bearing transmembrane cargos. This hypothesis was tested using chimaeric CD8 $\alpha$ (YV) as well as Frizzled4 (VV), and both depend on GRASP55 and GRASP65 for their transport to the plasma membrane [25]. This seems to be contrary to the result mentioned above where interfering with GRASP function inhibits Golgi stacking and increases CD8 transport to the plasma membrane [37]. Depletion of GRASP65 led to these cargos being retained in the ERES, whereas GRASP55 was seemingly required for their transport through the Golgi [25]. Their N-terminal domains seem to be involved in this interaction.

\section{GRASPs in unconventional protein secretion}

As mentioned above, depletion or knockout of GRASPs in many cell types results in neither a loss of Golgi stacking nor inhibition of bulk anterograde transport. In addition, GRASP is abundant on Golgi apparatuses where no stack structure is present, such as Drosophila follicle cells [5,40]. Furthermore, the mitotic phosphorylation of the SPR C-terminal domain seems limited to mammalian species and does not appear to take place in lower organisms, although this remains to be investigated in more detail. These observations imply that GRASPs may have additional cellular functions, which were investigated by generating GRASP mutants in Dictyostelium and Drosophila.

Classical ER-Golgi-plasma-membrane secretion, as outlined by the Nobel prize winner George Palade, predicts that only signal-peptide-containing proteins can be secreted in the extracellular medium. However, a small but significant pool of extracellular secreted proteins exists that are devoid of a signal peptide. These proteins are synthesized in the cytoplasm away from the membranes of the classical secretory pathway and include IL-1 $\beta$ (interleukin-1 $\beta$ ), galectin, FGF2 (fibroblast growth factor 2) and others [82].

Interestingly, the removal of the single gene encoding GRASP in Dictyostelium, grpA, revealed a role for this protein in unconventional secretion of a cellular non-membrane-associated factor, AcbA. Upon GABA ( $\gamma$-aminobutyric acid) induction, AcbA is produced in the cytoplasm of Dictyostelium prespore cells and is released in the extracellular medium, where it binds to a specific receptor and elicits signalling-promoting spore development [17]. Release of AcbA is blocked in grpA-null cells and AcbA-containing vesicles accumulate just beneath the plasma membrane, suggesting a role for grpA in the unconventional secretion of this cytoplasmic protein $[17,83]$. A similar role has recently been proved for Grh1 in $S$. cerevisiae and $P$. pastoris in the unconventional secretion of Acb1 in both yeasts $[79,80]$. The role of GRASPs in these processes remains to be carefully defined and has led to a number of models [82,84] (see below), including a role for GRASP at the plasma membrane.

A role for GRASP at the plasma membrane has recently been identified in relation to another type of unconventional secretion. Indeed, transmembrane proteins can also escape the classical secretory pathway and bypass the Golgi before being deposited to the plasma membrane [82,85]. In this regard, we have recently shown that dGRASP is necessary for Golgi bypass of the $\alpha$ PS1 subunit of integrins at very specific stages of Drosophila development during epithelial remodelling. This integrin subunit is then transported to the basal plasma membrane of epithelial cells in a dGRASP-dependent manner, but is insensitive to Brefeldin A treatment and the loss of syntaxin 5 [40]. Consequently, in Drosophila mutants for dGRASP, integrins are not properly deposited, and some epithelia such as the wing and follicular epithelium covering the oocyte are strongly disorganized. However, classical anterograde transport as a whole is not affected, in agreement with the lack of a role for GRASPs in general anterograde transport $[40,86]$.

Although different in the nature of its substrate and the type of secretion, it is remarkable that GRASP, a bona fide Golgi protein, exhibits an additional and novel developmentally controlled function both in Dictyostelium and Drosophila epithelium remodelling. Whether mammalian GRASPs have similar nonGolgi functions remains to be shown.

\section{GENE EXPRESSION REGULATION}

The majority of work analysing GRASP function has been at the biochemical, or cellular, level and the regulation of GRASP at the genetic level has gone mostly overlooked. However, there is evidence to suggest that gene and protein expression, as well as differential-splicing of GRASPs, can occur that could potentially explain the different roles outlined above (Figure 4).

First, the analysis of the intron/exon boundaries of different organisms reveals that the Grasp gene contains introns, so in principle it could be differentially spliced. Furthermore, their number has increased as evolution has passed. S. cerevisiae and $D$. discoideum have none; D. melanogaster has one; and most other higher organisms, such as Danio rerio, Mus musculus, Rattus norvegicus and Homo sapiens, have eight or nine perhaps reflecting a higher capacity for splicing events.

The human GRASP65 and GRASP55 genes display 28 and 13 different splice variants that encode nine and three proteins respectively (unpublished work using the Ensembl database). Some of these variants lack Gly ${ }^{2}$, suggesting that these isoforms could perhaps have a different molecular function to that of the characterized GRASP65 and GRASP55.

Regarding GRASP gene-expression modulation, the transcript levels of GRASP55 and GRASP65 have also been shown to be modulated. For instance, it has been shown that over the course of epithelial polarization and maturation in $\mathrm{CaCo}-2$ cells, a process that lasts 1-2 weeks, GRASP65 and GRASP55 mRNA levels are relatively high at the beginning and steadily decline towards the end [87]. This suggests that any function these proteins may have in this process is likely to occur at the early stages of epithelial polarization, for instance in the establishment of an epithelium.

Similarly, during rearrangement of the Drosophila follicular epithelium, dGrasp mRNA expression was shown to be upregulated and targeted in a developmentally controlled manner $[40,88]$. It has been demonstrated that the dGrasp gene can be 


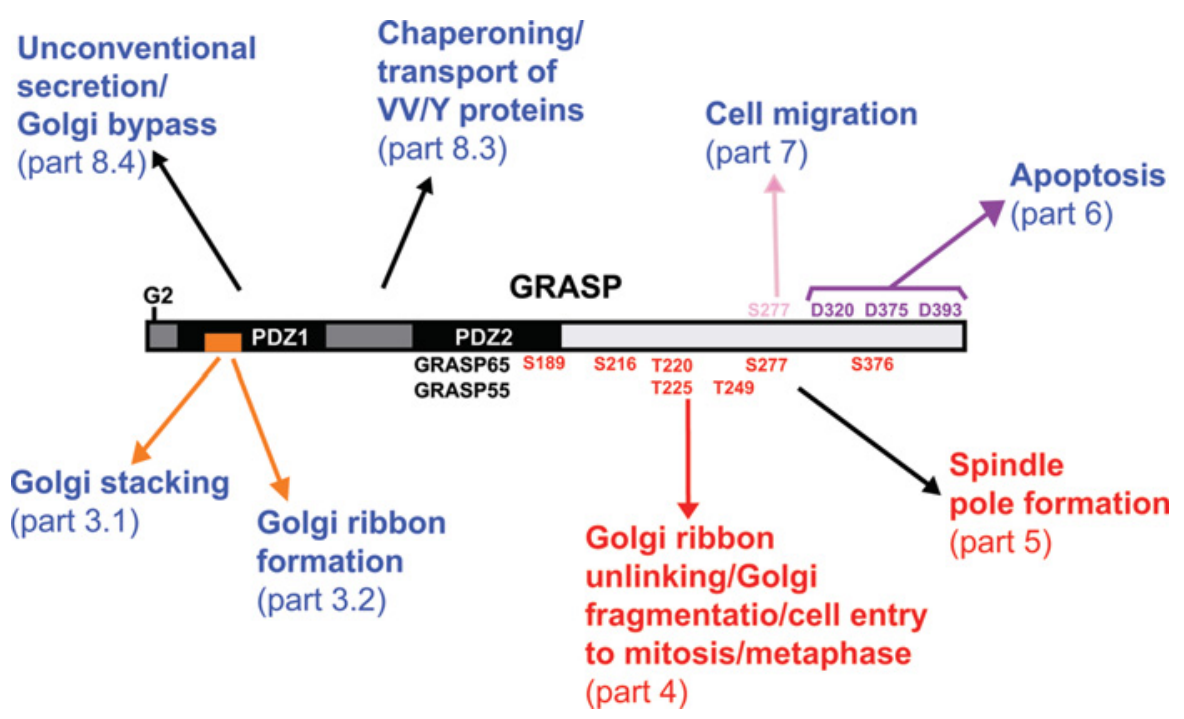

Figure 4 Multiple roles of GRASPs

The blue captions correspond to GRASP roles in interphase. The orange block represents the trans-oligomerization domain that plays a role in Golgi ribbon formation and perhaps in Golgi stacking The chaperoning/transport is proposed to involve the N-terminal half, whereas the GRASP role in apoptosis (pink arrow) and cell migration (violet arrow) involves amino acid residues situated in the C-terminal half. The red captions correspond to GRASP roles in mitosis and the phosphorylated serine residues and threonine residues are indicated in red.

very dynamically regulated. Given that the up-regulated $d$ Grasp mRNA gives rise to a polypeptide that ectopically localizes to the plasma membrane, it may suggest that this pool of upregulated dGrasp transcript encodes a protein that possesses different properties or varies in its amino acid sequence.

In this regard, the malarial parasite $P$. falciparum has two forms of GRASP. GRASP1 shares many molecular characteristics with the mammalian GRASPs, such as N-myristoylation, whereas GRASP2, a splice variant of GRASP1, does not use myristoylation but depends on its hydrophobic N-terminus to attach to membranes [19,22]. Curiously, both GRASP1 and GRASP2 target the Golgi membrane, so the reason for expressing these two isoforms is largely unexplained. However, the observation itself indicates that splicing of the GRASP gene can occur and leads to species with different biochemical properties and perhaps different functions. The potential human forms of GRASP55 and GRASP65 lacking Gly $^{2}$ might use the same membrane anchoring as $P$. falciparum GRASP2.

Lastly, although the levels or splicing of GRASPs have not been shown to be altered in cancers, GRASP65 is present on a region of chromosome 3 known for harbouring high tumoursuppressor genes [15]. The GRASP55 gene is present within a 'hot spot' known for loss of heterozygosity and/or homozygous loss in major epithelial cancers. This may mean that levels of GRASP65 could be down-regulated in cancers, something that has not been tested yet.

\section{CONCLUSIONS}

The surprisingly large number of roles for GRASPs were not anticipated when GRASP65 was first discovered 13 years ago. Many of the roles of the mammalian proteins, however, reflect the fate or behaviour of the Golgi complex in a number of physiological situations: Golgi stacking and Golgi ribbon formation, Golgi breakdown during mitosis and apoptosis, Golgi re-orientation to face the leading-edge of migrating cells and transport/chaperoning of $\mathrm{VV}$ proteins. However, in organisms present from earlier stages of evolution, GRASPs seem to have non-Golgi functions, perhaps at the plasma membrane. The challenge remains to establish whether those non-Golgi functions also exist for mammalian GRASPs.

Furthermore, a finer analysis of the different GRASP domains is needed, perhaps through the three-dimensional resolution structure of both the GRASP domain, but also the non-conserved C-terminus half. Establishing whether mammalian GRASPs can be replaced by a more ancient version of the protein and vice versa would provide a complementary approach. Lastly, study of the GRASP gene-expression pattern and regulation might provide clues on how these proteins can have so many facets.

\section{ACKNOWLEDGEMENTS}

We thank our colleagues in the field for helpful and lively discussions, in particular Adam Linstedt (Carnegie Mellon, Pittsburg, PA, U.S.A) for critically reading the manuscript and providing useful comments prior to submission. We thank Adrian Oprins for the illustration in Figure 1.

\section{FUNDING}

F.V. is funded by the Nederlandse Organisatie voor Wetenschappelijke Onderzoek (NOW) [Zon-MW TOP subsidie 912.080.24], and A.G. by ESF EuroMEMBRANE programme managed by NWO [CW 700.58.702]

\section{REFERENCES}

1 Rabouille, C., Misteli, T., Watson, R. and Warren, G. (1995) Reassembly of Golgi stacks from mitotic Golgi fragments in a cell-free system. J. Cell Biol. 129, 605-618

2 Rambourg, A. and Clermont, Y. (1997) Three-dimensional structure of the Golgi apparatus in mammalian cells. In The Golgi Apparatus (Berger, E. G. and Roth, J., eds), pp. 37-61, Birkhauser Verlag, Basel

3 Lowe, M., Nakamura, N. and Warren, G. (1998) Golgi division and membrane traffic. Trends Cell Biol. 8, 40-44

4 Kondylis, V. and Rabouille, C. (2003) A novel role for dp115 in the organization of tER sites in Drosophila. J. Cell Biol. 162, 185-198

5 Kondylis, V. and Rabouille, C. (2009) The Golgi apparatus: Iessons from Drosophila. FEBS Lett. 583, 3827-3838 
6 Levi, S. K., Bhattacharyya, D., Strack, R. L., Austin, II, J. R. and Glick, B. S. (2010) The yeast GRASP Grh1 colocalizes with COPII and is dispensable for organizing the secretory pathway. Traffic 11, 1168-1179

7 Cluett, E. B. and Brown, W. J. (1992) Adhesion of Golgi cisternae by proteinaceous interactions: intercisternal bridges as putative adhesive structures. J. Cell Sci. 103, 773-784

8 Slusarewicz, P., Nilsson, T., Hui, N., Watson, R. and Warren, G. (1994) Isolation of a matrix that binds medial Golgi enzymes. J. Cell Biol. 124, 405-413

9 Nakamura, N., Rabouille, C., Watson, R., Nilsson, T., Hui, N., Slusarewicz, P., Kreis, T. E. and Warren, G. (1995) Characterization of a cis-Golgi matrix protein, GM130. J. Cell Biol. 131, 1715-1726

10 Warren, G. (1993) Membrane partitioning during cell division. Annu. Rev. Biochem. 62 323-348

11 Rabouille, C. and Jokitalo, E. (2003) Golgi apparatus partitioning during cell division. Mol. Membr. Biol. 20, 117-127

12 Misteli, T. and Warren, G. (1994) COP-coated vesicles are involved in the mitotic fragmentation of Golgi stacks in a cell-free system. J. Cell Biol. 125, 269-282

13 Barr, F. A., Puype, M., Vandekerckhove, J. and Warren, G. (1997) GRASP65, a protein involved in the stacking of Golgi cisternae. Cell 91, 253-262

14 Shorter, J., Watson, R., Giannakou, M. E., Clarke, M., Warren, G. and Barr, F. A. (1999) GRASP55, a second mammalian GRASP protein involved in the stacking of Golgi cisternae in a cell-free system. EMBO J. 18, 4949-4960

15 Protopopov, A., Kashuba, V., Zabarovska, V. I., Muravenko, 0. V., Lerman, M. I., Klein, G. and Zabarovsky, E. R. (2003) An integrated physical and gene map of the 3.5-Mb chromosome 3p21.3 (AP20) region implicated in major human epithelial malignancies. Cancer Res. 63, 404-412

16 Shorter, J. and Warren, G. (1999) A role for the vesicle tethering protein, p115, in the post-mitotic stacking of reassembling Golgi cisternae in a cell-free system. J. Cell Biol. 146, $57-70$

17 Kinseth, M. A., Anjard, C., Fuller, D., Guizzunti, G., Loomis, W. F. and Malhotra, V. (2007) The Golgi-associated protein GRASP is required for unconventional protein secretion during development. Cell 130, 524-534

18 Katinka, M. D., Duprat, S., Cornillot, E., Metenier, G., Thomarat, F., Prensier, G., Barbe, V., Peyretaillade, E., Brottier, P., Wincker, P. et al. (2001) Genome sequence and gene compaction of the eukaryote parasite Encephalitozoon cuniculi. Nature 414, 450-453

19 Struck, N. S., Herrmann, S., Langer, C., Krueger, A., Foth, B. J., Engelberg, K., Cabrera, A. L., Haase, S., Treeck, M., Marti, M. et al. (2008) Plasmodium falciparum possesses two GRASP proteins that are differentially targeted to the Golgi complex via a higher- and lower-eukaryote-like mechanism. J. Cell Sci. 121, 2123-2129

20 Kondylis, V., Spoorendonk, K. M. and Rabouille, C. (2005) dGRASP Iocalization and function in the early exocytic pathway in Drosophila S2 cells. Mol. Biol. Cell 16, 4061-4072

21 Yelinek, J. T., He, C. Y. and Warren, G. (2009) Ultrastructural study of Golgi duplication in Trypanosoma brucei. Traffic 10, 300-306

22 Struck, N. S., de Souza Dias, S., Langer, C., Marti, M., Pearce, J. A., Cowman, A. F. and Gilberger, T. W. (2005) Re-defining the Golgi complex in Plasmodium falciparum using the novel Golgi marker PfGRASP. J. Cell Sci. 118, 5603-5613

23 Behnia, R., Barr, F. A., Flanagan, J. J., Barlowe, C. and Munro, S. (2007) The yeast orthologue of GRASP65 forms a complex with a coiled-coil protein that contributes to ER to Golgi traffic. J. Cell Biol. 176, 255-261

24 Marra, P., Maffucci, T., Daniele, T., Tullio, G. D., Ikehara, Y., Chan, E. K., Luini, A., Beznoussenko, G., Mironov, A. and de Matteis, M. A. (2001) The GM130 and GRASP65 Golgi proteins cycle through and define a subdomain of the intermediate compartment. Nat. Cell Biol. 3, 1101-1113

25 D'Angelo, G., Prencipe, L., lodice, L., Beznoussenko, G., Savarese, M., Marra, P., di Tullio, G., Martire, G., de Matteis, M. A. and Bonatti, S. (2009) GRASP65 and GRASP55 sequentially promote the transport of C-terminal valine-bearing cargos to and through the Golgi complex. J. Biol. Chem. 284, 34849-34860

26 Wang, Y., Satoh, A. and Warren, G. (2005) Mapping the functional domains of the Golgi stacking factor GRASP65. J. Biol. Chem. 280, 4921-4928

27 Ramirez, I. B. and Lowe, M. (2009) Golgins and GRASPs: holding the Golgi together. Semin. Cell Dev. Biol. 20, 770-779

28 Barr, F. A., Nakamura, N. and Warren, G. (1998) Mapping the interaction between GRASP65 and GM130, components of a protein complex involved in the stacking of Golgi cisternae. EMBO J. 17, 3258-3268

29 Sutterlin, C., Polishchuk, R., Pecot, M. and Malhotra, V. (2005) The Golgi-associated protein GRASP65 regulates spindle dynamics and is essential for cell division. Mol. Biol. Cell 16, 3211-3222

30 Puthenveedu, M. A., Bachert, C., Puri, S., Lanni, F. and Linstedt, A. D. (2006) GM130 and GRASP65-dependent lateral cisternal fusion allows uniform Golgi-enzyme distribution. Nat. Cell Biol. 8, 238-248
31 Xiang, Y. and Wang, Y. (2010) GRASP55 and GRASP65 play complementary and essential roles in Golgi cisternal stacking. J. Cell Biol. 188, 237-251

32 Short, B., Preisinger, C., Korner, R., Kopajtich, R., Byron, 0. and Barr, F. A. (2001) A GRASP55-rab2 effector complex linking Golgi structure to membrane traffic. J. Cell Biol. 155, 877-883

33 Wang, Y., Seemann, J., Pypaert, M., Shorter, J. and Warren, G. (2003) A direct role for GRASP65 as a mitotically regulated Golgi stacking factor. EMBO J. 22, 3279-3290

34 Sengupta, D., Truschel, S., Bachert, C. and Linstedt, A. D. (2009) Organelle tethering by a homotypic PDZ interaction underlies formation of the Golgi membrane network. J. Cell Biol. 186, 41-55

35 Bachert, C. and Linstedt, A. D. (2010) Dual anchoring of the GRASP membrane tether promotes trans pairing. J. Biol. Chem. 285, 16294-16301

36 Kay, B. K., Williamson, M. P. and Sudol, M. (2000) The importance of being proline: the interaction of proline-rich motifs in signaling proteins with their cognate domains. FASEB J. 14, 231-241

37 Wang, Y., Wei, J. H., Bisel, B., Tang, D. and Seemann, J. (2008) Golgi cisternal unstacking stimulates COPI vesicle budding and protein transport. PLOS One 3, e1647

38 Duran, J. M., Kinseth, M., Bossard, C., Rose, D. W., Polishchuk, R., Wu, C. C., Yates, J., Zimmerman, T. and Malhotra, V. (2008) The role of GRASP55 in Golgi fragmentation and entry of cells into mitosis. Mol. Biol. Cell 19, 2579-2587

39 Feinstein, T. N. and Linstedt, A. D. (2008) GRASP55 regulates Golgi ribbon formation. Mol. Biol. Cell 19, 2696-2707

40 Schotman, H., Karhinen, L. and Rabouille, C. (2008) dGRASP-mediated noncanonical integrin secretion is required for Drosophila epithelial remodeling. Dev. Cell 14, 171-182

41 Barr, F. A., Preisinger, C., Kopajtich, R. and Korner, R. (2001) Golgi matrix proteins interact with p24 cargo receptors and aid their efficient retention in the Golgi apparatus. J. Cell Biol. 155, 885-891

42 Tang, D., Yuan, H. and Wang, Y. (2010) The role of GRASP65 in Golgi cisternal stacking and cell cycle progression. Traffic 11, 827-842

43 Sengupta, D. and Linstedt, A. D. (2010) Mitotic inhibition of GRASP65 organelle tethering involves Polo-like kinase 1 (PLK1) phosphorylation proximate to an internal PDZ ligand. J. Biol. Chem. doi:10. 1074/jbc.M110.189449

44 Lin, C. Y., Madsen, M. L., Yarm, F. R., Jang, Y. J., Liu, X. and Erikson, R. L. (2000) Peripheral Golgi protein GRASP65 is a target of mitotic polo-like kinase (PIk) and Cdc2. Proc. Natl. Acad. Sci. U.S.A. 97, 12589-12594

45 Preisinger, C., Korner, R., Wind, M., Lehmann, W. D., Kopajtich, R. and Barr, F. A. (2005) Plk1 docking to GRASP65 phosphorylated by Cdk1 suggests a mechanism for Golgi checkpoint signalling. EMBO J. 24, 753-765

46 Sutterlin, C., Lin, C. Y., Feng, Y., Ferris, D K., Erikson, R. L. and Malhotra, V. (2001) Polo-like kinase is required for the fragmentation of pericentriolar Golgi stacks during mitosis. Proc. Natl. Acad. Sci. U.S.A. 98, 9128-9132

47 Yoshimura, S., Yoshioka, K., Barr, F. A., Lowe, M., Nakayama, K., Ohkuma, S. and Nakamura, N. (2005) Convergence of cell cycle regulation and growth factor signals on GRASP65. J. Biol. Chem. 280, 23048-23056

48 Jesch, S. A., Lewis, T. S., Ahn, N. G. and Linstedt, A. D. (2001) Mitotic phosphorylation of Golgi reassembly stacking protein 55 by mitogen-activated protein kinase ERK2. Mol. Biol. Cell 12, 1811-1817

49 Shaul, Y. D. and Seger, R. (2006) ERK1c regulates Golgi fragmentation during mitosis. J. Cell Biol. 172, 885-897

50 Lowery, D. M., Clauser, K. R., Hjerrild, M., Lim, D., Alexander, J., Kishi, K., Ong, S. E., Gammeltoft, S., Carr, S. A. and Yaffe, M. B. (2007) Proteomic screen defines the Polo-box domain interactome and identifies Rock2 as a Plk1 substrate. EMBO J. 26, 2262-2273

51 Rabouille, C. and Kondylis, V. (2007) Golgi ribbon unlinking: an organelle-based G2/M checkpoint. Cell Cycle 6, 2723-2729

52 Persico, A., Cervigni, R. I., Barretta, M. L. and Colanzi, A. (2009) Mitotic inheritance of the Golgi complex. FEBS Lett. 583, 3857-3862

53 Sutterlin, C., Hsu, P., Mallabiabarrena, A. and Malhotra, V. (2002) Fragmentation and dispersal of the pericentriolar Golgi complex is required for entry into mitosis in mammalian cells. Cell 109, 359-369

54 Feinstein, T. N. and Linstedt, A. D. (2007) Mitogen-activated protein kinase kinase 1-dependent Golgi unlinking occurs in $\mathrm{G} 2$ phase and promotes the G2/M cell cycle transition. Mol. Biol. Cell 18, 594-604

55 Norman, T. C., Smith, D. L., Sorger, P. K., Drees, B. L., O'Rourke, S. M., Hughes, T. R., Roberts, C. J., Friend, S. H., Fields, S. and Murray, A. W. (1999) Genetic selection of peptide inhibitors of biological pathways. Science 285, 591-595

56 Thyberg, J. and Moskalewski, S. (1999) Role of microtubules in the organization of the Golgi complex. Exp. Cell Res. 246, 263-279

57 Reilein, A. R., Serpinskaya, A. S., Karcher, R. L., Dujardin, D. L., Vallee, R. B. and Gelfand, V. I. (2003) Differential regulation of dynein-driven melanosome movement. Biochem. Biophys. Res. Commun. 309, 652-658 
58 Corthesy-Theulaz, I., Pauloin, A. and Pfeffer, S. R. (1992) Cytoplasmic dynein participates in the centrosomal localization of the Golgi complex. J. Cell Biol. 118, 1333-1345

59 Cole, N. B., Sciaky, N., Marotta, A., Song, J. and Lippincott-Schwartz, J. (1996) Golgi dispersal during microtubule disruption: regeneration of Golgi stacks at peripheral endoplasmic reticulum exit sites. Mol. Biol. Cell 7, 631-650

60 Miller, P. M., Folkmann, A. W., Maia, A. R., Efimova, N., Efimov, A. and Kaverina, I. (2009) Golgi-derived CLASP-dependent microtubules control Golgi organization and polarized trafficking in motile cells. Nat. Cell Biol. 11, 1069-1080

61 Sutterlin, C. and Colanzi, A. (2010) The Golgi and the centrosome: building a functional partnership. J. Cell Biol. 188, 621-628

62 Wei, J. H. and Seemann, J. (2009) Spindle-dependent partitioning of the Golgi ribbon. Commun. Integr. Biol. 2, 406-407

63 Kodani, A. and Sutterlin, C. (2008) The Golgi protein GM130 regulates centrosome morphology and function. Mol. Biol. Cell 19, 745-753

64 Sesso, A., Fujiwara, D. T., Jaeger, M., Jaeger, R., Li, T. C., Monteiro, M. M., Correa, H., Ferreira, M. A., Schumacher, R. I., Belisario, R. I. et al. (1999) Structural elements common to mitosis and apoptosis. Tissue Cell 31, 357-371

65 Maag, R. S., Hicks, S. W. and Machamer, C E. (2003) Death from within: apoptosis and the secretory pathway. Curr. Opin. Cell Biol. 15, 456-461

66 Hengartner, M. 0. (2000) The biochemistry of apoptosis. Nature 407, 770-776

67 Walker, A., Ward, C., Sheldrake, T. A., Dransfield, I., Rossi, A. G., Pryde, J. G. and Haslett, C. (2004) Golgi fragmentation during Fas-mediated apoptosis is associated with the rapid loss of GM130. Biochem. Biophys. Res. Commun. 316, 6-11

68 Chiu, R., Novikov, L., Mukherjee, S. and Shields, D. (2002) A caspase cleavage fragment of p115 induces fragmentation of the Golgi apparatus and apoptosis. J. Cell Biol. 159, 637-648

69 Lowe, M., Lane, J. D., Woodman, P. G. and Allan, V. J. (2004) Caspase-mediated cleavage of syntaxin 5 and giantin accompanies inhibition of secretory traffic during apoptosis. J. Cell Sci. 117, 1139-1150

70 Mancini, M., Machamer, C. E., Roy, S., Nicholson, D. W., Thornberry, N. A., Casciola-Rosen, L. A. and Rosen, A. (2000) Caspase-2 is localized at the Golgi complex and cleaves golgin-160 during apoptosis. J. Cell Biol. 149, 603-612

71 Lane, J. D., Lucocq, J., Pryde, J., Barr, F. A., Woodman, P. G., Allan, V. J. and Lowe, M. (2002) Caspase-mediated cleavage of the stacking protein GRASP65 is required for Golgi fragmentation during apoptosis. J. Cell Biol. 156, 495-509

72 Nakagomi, S., Barsoum, M. J., Bossy-Wetzel, E., Sutterlin, C., Malhotra, V. and Lipton, S. A. (2008) A Golgi fragmentation pathway in neurodegeneration. Neurobiol. Dis. 29 221-231

73 Preisinger, C. and Barr, F. A. (2001) Signaling pathways regulating Golgi structure and function. Sci. STKE 106, pe38

Received 22 September 2010/14 October 2010; accepted 18 October 2010

Published on the Internet 14 January 2011, doi:10.1042/BJ20101540
74 Yadav, S., Puri, S. and Linstedt, A. D. (2009) A primary role for Golgi positioning in directed secretion, cell polarity, and wound healing. Mol. Biol. Cell 20, 1728-1736

75 Bisel, B., Wang, Y., Wei, J. H., Xiang, Y., Tang, D., Miron-Mendoza, M., Yoshimura, S. Nakamura, N. and Seemann, J. (2008) ERK regulates Golgi and centrosome orientation towards the leading edge through GRASP65. J. Cell Biol. 182, 837-843

76 Spang, A. (2009) On vesicle formation and tethering in the ER-Golgi shuttle. Curr. Opin. Cell Biol. 21, 531-536

77 Bard, F., Casano, L., Mallabiabarrena, A., Wallace, E., Saito, K., Kitayama, H., Guizzunti, G., Hu, Y., Wendler, F., Dasgupta, R et al. (2006) Functional genomics reveals genes involved in protein secretion and Golgi organization. Nature $\mathbf{4 3 9}, 604-607$

78 Wendler, F., Gillingham, A. K., Sinka, R., Rosa-Ferreira, C., Gordon, D. E., Franch-Marro X., Peden, AA., Vincent, J. P. and Munro, S. (2010) A genome-wide RNA interference screen identifies two novel components of the metazoan secretory pathway. EMBO J. 29 , 304-314

79 Duran, J. M., Anjard, C., Stefan, C., Loomis, W. F. and Malhotra, V. (2010) Unconventional secretion of Acb1 is mediated by autophagosomes. J. Cell Biol. 188, 527-536

80 Manjithaya, R., Anjard, C., Loomis, W. F. and Subramani, S. (2010) Unconventional secretion of Pichia pastoris Acb1 is dependent on GRASP protein, peroxisomal functions, and autophagosome formation. J. Cell Biol. 188, 537-546

81 Kuo, A., Zhong, C., Lane, W. S. and Derynck, R. (2000) Transmembrane transforming growth factor- $\alpha$ tethers to the PDZ domain-containing, Golgi membrane-associated protein p59/GRASP55. EMBO J. 19, 6427-6439

82 Nickel, W. and Rabouille, C. (2009) Mechanisms of regulated unconventional protein secretion. Nat Rev Mol Cell Biol. 10, 148-155

83 Cabral, M., Anjard, C., Malhotra, V., Loomis, W. F. and Kuspa, A. (2010) Unconventional secretion of AcbA in Dictyostelium discoideum through a vesicular intermediate. Eukaryot. Cell 9, 1009-1017

84 Levi, S. K. and Glick, B. S. (2007) GRASPing unconventional secretion. Cell 130 407-409

85 Grieve, A. G. and Rabouille, C. (2010) Golgi bypass: skirting around the heart of classical secretion. In The Golgi Book: Cold Spring Harbor Perspectives in Biology (Warren, G. and Rothman, J., eds), Cold Spring Harbor Laboratory Press, Cold Spring Harbor

86 Nickel, W. (2010) Pathways of unconventional protein secretion. Curr. Opin. Biotechnol. 21, 621-626

87 Halbleib, J. M., Saaf, AM., Brown, P. 0. and Nelson, W. J. (2007) Transcriptional modulation of genes encoding structural characteristics of differentiating enterocytes during development of a polarized epithelium in vitro. Mol. Biol. Cell 18, 4261-4278

88 Schotman, H., Karhinen, L. and Rabouille, C. (2009) Integrins mediate their unconventional, mechanical-stress-induced secretion via RhoA and $\mathrm{PINCH}$ in Drosophila. J. Cell Sci. 122, 2662-2672 